\title{
Medical information Recommendation based on Scenario Model and User Preference in Mobile Internet Era
}

\author{
Yang Fuhao, Lin Shaofu \\ Beijing University of Technology, Beijing100124, China
}

Keywords: Medical Information recommendation; Scene Model; User Preference; Mobile Client

\begin{abstract}
The optimization of the mobile web environment and the popularity of smart phones and other mobile devices enable users to access to Internet at any time and any place, not restricted by the location. By smart phone, people can access to medical information more convenient, but in fact, users really find their required information and begin to pay attention, still need a longer operating path. In order to solve the users' pain points, we propose a recommendation system architecture that combines the scene model to enable users to personalized medical information, at the right time, right place, convenient access to the medical information he, does not need to consume a lot of energy to make their required medical information.
\end{abstract}

\section{Introduction}

Under the development of society, people's demand for medical resources is growing, and short-term shortage of medical resources is an objective fact. With the current development of the mobile Internet tide, the various types of APP on the phone quickly have a large number of users and the user's online time. The patient can get better help through the medical information recommended APP alternative part of the medical resources. But the current medical applications lack the user's personalization, this article mainly aims to analyze and discuss the viable solutions of these problems.

\section{The design of personalized mobile medical information client}

This section mainly around the design and the plan of the recommendation system to do a detailed description on the practical implementation, including the design of the main process, system architecture, application scenarios, etc., however, the main aspect is the client, which as a key link of the medical information recommended system, plays an important role. All the information collection of the users and the environment is achieved through the client's function. Most of this article uses Android client as an example of clients' content. So the client development environment mentioned below is primarily a development environment for Android.

Client operating environment: Android

Client development environment: Windows; IntelliJ IDEA; Java; C + +

Service operating environment: CentOS; MySQL Cluster. Hadoop Cluster (Baidu cloud storage BCS)

Server development environment: Linux; C + +; Java; PHP

2.1 The design of personalized recommendation system

Can the recommendations function achieve the desired effect that needs to trigger users' participation feedback continuously after a long time, let the users own correct our recommendation 
results by themselves, at the same time, help us to improve our recommendation algorithm, different users' feedback will give different weights to the appropriate medical information, thus affect the recommendation result.

Back-end mainly uses the collaborative filtering recommendation algorithm method (ItemCF), which mainly is divided into the Online Item CF and offline Item CF, both of them are according to the WEB interface to access to the front-end application. The online ItemCF, which is used primarily in response to the instant request on the line, and handles the changing data at any time, and the amount of calculation is not too large to cause the response time to be extended. The off-line ItemCF is used to handle a large number of user behavior logs, which can be handled through cloud computing to manage time-consuming calculations. Both online and offline ItemCF calculations require the basic similarity matrix as the basis of the algorithm. The purpose of the calculation similarity matrix is to calculate the pairwise similarity, of the medical information of the rolls to every scene. It is through the method with the heat of weighted association rules. The reason why we introduce heat weight is that, naturally, high heat press will associated with most of the medical information, so that it is necessary to punish this kind of phenomenon.

One of key points of the Algorithm is the selection of neighbor, which involves the actual workload. At the same time, because different guidance nodes have different importance in forecast, should choose the most trustworthy neighbor node to predict. On the other hand according to the algorithm to calculate the user's preferences model (vector) as the User Profile, therefore, according to the neighbor node (Neighbor) and basic similarity matrix to generate the initial list of recommendation, utilizing User Profile to filter bad case.

The related calculations of the Item CF algorithm are as follows:

$$
\begin{aligned}
& r_{u i}^{\prime}=r_{u i}-u-b_{u}-b_{i} \\
& u=\frac{\sum_{u, i \in K} r_{u i}}{|N(u, i)|} \quad b_{i}=\frac{\sum_{u \in R(i)}\left(r_{u i}-u\right)}{\lambda+|R(i)|} \quad b_{u}=\frac{\sum_{i \in R(u)}\left(r_{u i}-u-b_{i}\right)}{\lambda+|R(u)|} \\
& w_{i j}=\frac{\left(\sum_{u \in U(i, j)} r_{u i}^{\prime} * r_{u j}^{\prime}\right) *(|U(i, j)|-1)}{\sqrt{\sum_{u \in U(i, j)} r_{u i}^{\prime 2} * \sum_{u \in U(i, j)} r_{u j}^{\prime 2}} *(|U(i, j)|-1+\lambda)} \\
& r_{u i}^{\text {predict }}=b_{i}+\sum_{j \in S^{k}(i ; u)} r_{u j}^{\prime} w_{j i}
\end{aligned}
$$

2.2 The analysis of scenario awareness personalized medical information recommendation system

The recommendation of the mobile platform itself differs from the traditional way in different aspect as follows.

(1) Users of the mobile platform can use the client to access the recommendation system at any place and time. The recommended results of mobile users should be more closely related to situational information such as time and place. Situational awareness of mobile recommendations is higher than the traditional recommended in real-time requirement.

(2) The discreteness of the recommendation of the mobile platform, the mobile platform has a lot of no or weak network connection; we need to consider the problem of calculation of offline or 
lightweight network dependent calculation.

(3) Mobile device have small screen, limited display space, inconvenient operation , so the recommendations need to make corresponding changes to adapt to the situation, such as recommend results should be refining, and minimize the frequency of prompting the users to click the small button. For medical information applications, we can even consider presenting our recommendations in the form of sound inserts. For the situational requirements of mobile platforms recommendation, in conjunction with the scenario list, we designed the personalized medical information list recommendations. The difference between the process and the separated scene recommendation is that the result of combined with the users' personality, finally, the list, which is displayed to the user is likely to fit their current status of medical information, each scene medical information list contains a personalized medical information list, which is generate according to the user's information and browsing habits.

\section{The implementation of mobile medical information client module}

The followings take Android client as an example to explain he specific design of mobile medical information clients.

As mobile medical information client, firstly, should have the ordinary medical information recommendation function, and a typical personalized medical information recommendation, at least should have the following basic functional category: medical information selection, medical information broadcasting, medical information retrieval, personalized settings and log collection. Quickly retrieve medical information is the user's fundamental demand, and is also the biggest pain points of us.

In order to satisfy the users' requirements, what we think firstly is through data mining, collaborative filtering to recommend for them, and users don't have to find medical information by themselves, the system directly provide the medical information what he might like. this is what the personalized recommendation function modules need to complete.

But such recommendations only according to the user's past medical information browsing behavior and retrieved information to mine the users' preferences, but it is still far away from the users' needs, They need the medical information which is more in line with their mood at this very moment, so combines the personalized recommendation with situational awareness, and opens out the scene function modules,. So that we can satisfy the users' personalized medical information selection demands on the basic of different scenarios.

Starting from the moment that the users have the demands of reading medical information to they see what they want, that is to say, from initiating the connection with the application server to a user received the push medical information and click, it would be better if there is no delay or slowness, it requires a powerful optimized network module to support.

\subsection{Personalized module}

Entry of off-line computation part of the personalized recommendation module is the client online resources web page, a "guess like" list of items, click the list item, will enter a list of daily recommended medical information, which contains the medical information lists that is based on users' operation information.

Online part mainly is about the function that the recommendation of personalized radio station, the users through the collection and classified operation of the personalized medical information trigger system computation to generate the user model, which optimizes the list of recommended medical information of the radio stations.

Firstly, to Correspond to the "guess you like" function on the client concretely, It demands to 
collect the user's operation information and login information, when the user on the client have any operation, such as click to enter one kind of the medical information operation, background threads will real-time record the user's operations, and feedback the information to the log platform at the right time. Every morning, the data mining module of the recommendation system will extract the log from the log platform, then calculate the offline model. The recommendation service will calculate the "recommended medical information today" based on the off-line model. Then through the recommended web application program interface (WEB API) feeds back to the online feedback system, after calculating in the online system, to generate the instant model feedback to the recommendation system. According to the real-time model, recommendation service returns the generated recommendation results (medical information list) to the client., When the user first launches the client the next morning, he will receive the recommended medical information list according to the recommendation interface.

\subsection{Scenario-based module}

Scenario-based module is mainly engaged in at the right time and place to provide users with the appropriate medical information, first of all, we need to have a big classified library of medical information, which contains the medical information what you will need and each of the category has a long list of medical information. On the client, there is a special scene module that is used to list the lists for the users, as for the users, the choice is very direct, is also very effective.

\section{The implementation of personalized scenario-based medical information recommendations}

Situational awareness is not achieved overnight, in order to distinguish the scenario as accurately as possible, we need to judge from the analysis of the user' characteristics and data mining in a long-term, at the same time, it demands the users to take part in the choice actively to optimize the algorithm.

\section{1 scenario recognition of fixed position and time}

In conjunction with the user's identity tag information, it is a continuous, relative and optimal process to give the recommendation of scenario medical information at a given time, This process requires a huge classified medical information library, based on the original medical information attribute tags, put most of the medical information into some certain scene classification artificially, and then according to the user's "click" and "browse" operation to check whether the accuracy of the medical information classification.

How to correspond to a particular scene at the right time? Firstly, dividing the time artificially, such as "Early morning", "Morning", "Afternoon", "Evening", "Night" and so on, and then, generating recommendations medical information according to what most people have seen belong to what kind of situation .Users can choose one of these categories they are interested in d to browse, at the same time, the user's choice has also become a meaningful feedback, the selected add positive ratings, the unselected add negative ratings, recommended system can optimize and modify the result of original artificial recommendation, which is based on a lot of similar feedback and summary and can form a self-learning system. The user's similar users will be more accurately recommended by the proposed algorithm, such as concrete collaborative filtering.

4.2The scenario-based medical information recommendations reminder of specific time

In order to improve the user's activity and speed up the optimization of our recommendation algorithm, we need to remind the users to listen to our scenario medical information at the right time, or when users forget our function, we will timely delivery to the user what he may want. Because only the user's browsing operation long enough can we improve the user model and get the medical information recommendations more accurate. The medical information that is 
recommended is new and is exactly what he likes, and it also fits his needs there and then. The whole function is what we called personalized, scenario-based medical information recommendations.

The core of this design is the optimization process of mining algorithms and the precision of push time. We via a large number of data mining algorithm to dig up the users who need the recommendation timely and their suitable medical information list, and then in the early morning every day, the recommended service uses cloud computing to generate the list of users and recommendation result, and then calls the API of the push system to generate a set of tasks, the next day, timing tasks will be send to the user's mobile device timely.

If there is a push task that the big medical information or the broadcasting which are needed to pushed to all user's embedded, we can cancel all of the settings of the timing personalized push task, which aims to reduce the disturbance to the users with too much push.

\section{Summary}

With the spread of mobile Internet devices such as smart phones and tablets great change have taken place in the way and scenario of medical information services for people. Through the medical information recommendation to solve the problem of medical treatment is not a bad way, but recommended effect is good or not in the practical which is always difficult to solve, the current domestic and foreign people all have no good solutions. This article presents a recommendation system architecture based on scenario model and cloud computing, which provides users with recommendations which are more in line with the actual needs of users.

\section{References}

[1] Badrul Sarwar, George Karypis, and Joseph Konstan. Item-based Collaborative Filtering Recommendation Algorithms. Proceedings of the 10th, pages 285-295,2001.

[2] Xiaoyuan Su and Taghi M. Khoshgoftaar. A Survey of Collaborative Filtering Techniques. Advances in Artificial Intelligence, 2009(Section 3):1-19, 2009.

[3] Robin Burke. Hybrid Recommender Systems: Survey and Experiments. User Modeling and User-Adapted Interaction, 12(4):331-370, 2002.

[4] G. Adomavicius and A. Tuzhilin. Toward the Next Generation of Recommender Systems: A Survey of the State-of-the-art and Possible Extensions. IEEE Transactions on Knowledge and Data Engineering, 17(6):734-749, June 2005.

[5] G.Shani, D. Heckerman, and R.I. Brafman. An MDP-Based Recommender System. J.Mach.Learn.Res, 2005.

[6] Bobadilla J, Ortega F, and Hernando A. Acollaborative filtering similarity measure based on singularities[J]. Information Processing \& Management, 2012, 48(2):204-217.

[7] Macqueen J. Some Methods for Classificationand Analysis of MultiVariate Observations[C]// In 5th Berkeley Symp. Math. Statist. Prob. 2015:281-297.

[8] Schafer J B, Dan F, Herlocker J, et al. Collaborative Filtering Recommender Systems[C]// 
The Adaptive Web, Methods and Strategies of Web Personalization. 2015:46-45.

[9] B. Sarwar, G. Karypis, J. Konstan, and J. Riedl. Item-based collaborative filtering recommendation algorithms. In WWW '01: Proceedings of the 10th international conference on World Wide Web, pages 285-295, New York, NY, USA, 2001, ACM.

[10] Schmidt S, Steele R, Dillon T S, et al. Fuzzy trust evaluation and credibility development in multi-agent systems[J]. Applied Soft Computing, 2007, 7(2):492-505.

[11] Yiming Yang, Pedersen J.P. A Comparative Study on Feature Selection in Text Categorization. Proceedings of the 14th International Conference on Machine Learning (ICML'97), 1997: 412-420.

[12] Sriram B, Fuhry D, Demir E, et al. Short text classification in twitter to improve information filtering[J]. Sigir Proceeding of International Acm Sigir Conference on Research \& Development in I, 2010:841-842.

[13] CHANG C C, LIN C J. LIBSVM: a library for support vector machines [J]. ACM Trans on intelligent Systems and Technology, 2011, 2(3): 27-56.

[14] M.D. Ekstrand, J.T. Riedl, J.A. Konstan. Collaborative filtering recommender systems[M].Foundation and Trends in Human-Computer Interaction, Now Publishers lnc.,2011.

[15] Zaharia M, Chowdhury M, Das T, et al. Resilient distributed datasets: A fault-tolerant abstraction for in-memory cluster computing[C]//Proceedings of the 9thUSENIX conference on Networked Systems Design and Implementation. USENIX Association, 2012: 2-2. 